\title{
Conocimiento Especializado de un Profesor de Matemáticas de Educación Primaria al Enseñar los Números Racionales
}

\section{Specialized Knowledge of One Primary Mathematics Teacher Teaching}

\author{
Rational Numbers
}

\author{
Nielka Rojas* \\ Pablo Flores** \\ José Carrillo***
}

\begin{abstract}
Resumen
Describimos el conocimiento especializado de un profesor de matemáticas experto de Educación Primaria, que enseña el tema de los números racionales a estudiantes de 11 a 12 años de edad. Realizamos un análisis de contenido de las transcripciones de las 21 sesiones de clase impartidas por el profesor, empleando un sistema de categorías de los subdominios de conocimiento del modelo de Conocimiento Especializado del Profesor de Matemáticas (MTSK). Ello nos permitió apreciar los conocimientos manifestados por el docente en su práctica, especialmente en tres subdominios, el conocimiento de los temas matemáticos, de las características del aprendizaje de las matemáticas y de la enseñanza de las matemáticas.
\end{abstract}

Palabras clave: Conocimiento Matemático para la Enseñanza. Conocimiento Especializado del Profesor de Matemáticas. Profesor Experto. Números Racionales. Estudio de Caso.

\begin{abstract}
We describe the specialized knowledge of an expert Primary Education math teacher while teaching the topic of rational numbers to students of 11-12 years old. Using a category system of the subdomains of the Mathematics Teacher's Specialized Knowledge model (MTSK), a content analysis of the transcripts of 21 classes taught was performed. This allowed us to appreciate the knowledge expressed by the teacher in his practice, especially in three subdomains: mathematical content knowledge, characteristics of the learning of mathematics, and knowledge of mathematics teaching.
\end{abstract}

Keywords: Mathematical Knowledge for Teaching. Mathematics Teacher Specialized Knowledge. Expert Teacher. Rational Numbers. Case Study.

\section{Introducción}

* Doctor en Matemáticas, Universidad de Granada (UGR), España. Profesor de la Escuela de Educación, Universidad Católica del Norte (UCN), Antofagasta, Chile. Dirección postal: Angamos 0610. Antofagasta, Chile. E-mail: nrojas03@ucn.cl.

** Licenciado en Matemáticas y Doctor en Matemáticas por la Universidad de Granada (UGR). Profesor Titular de Universidad de Didáctica de la Matemática, Universidad de Granada (UGR), Granada, España. Dirección postal: Facultad de de Educación, Campus Cartuja, 18071, Granada, España. E-mail: pflores@ugr.es.

*** Licenciado en Matemáticas y Doctor en Filosofía y Ciencias de la Educación por la Universidad de Sevilla (US). Profesor Catedrático de la Universidad de Didáctica de la Matemática, Universidad de Huelva (UHU), Huelva, España. Dirección postal: Facultad de Ciencias de La Educación, Campus El Carmen, Avenida 3 de Marzo, s/n, 21071, Huelva, España. E-mail: carrillo@uhu.es. 
Actualmente, uno de los problemas principales en la línea de investigación sobre la Formación de Profesores de Matemática es estudiar el conocimiento profesional del profesorado (ENGLISH, 2008), donde se analiza su naturaleza, las características que lo conforman, el grado de conocimiento matemático que tienen y han de tener los profesores para desarrollar su tarea docente. Aspectos reflejados en las distintas reuniones internacionales de Educación Matemática celebradas cada año y en las nuevas revistas de profesores que han ido surgiendo en el último tiempo.

Es evidente que existen inquietudes acerca del conocimiento y las habilidades del profesor y en cómo estas cuestiones repercuten en la tarea de enseñanza, correspondiendo a algunos de los fenómenos que se estudian en Educación Matemática (SCHOENFELD, 2010). El informe Teachers for Tomorrow's Schools (OCDE, 2001) que analiza los indicadores educativos desarrollados por la OCDE/UNESCO (World Education Indicators - WEI), sostiene que obtener información a un nivel micro es indispensable, ya que se requieren evidencias que provengan directamente del aula, como una vía para comprender los problemas reales de la educación (OCDE, 2001, p. 14). De este modo, contar con nuevas experiencias, identificadas a partir de la práctica de los propios profesores, puede llevar a comprender las distintas situaciones a las que se enfrentan en su labor docente (RIBEIRO, 2009).

La actuación del profesor está influenciada por el conocimiento profesional, que le permite diseñar, aplicar, actuar frente a las respuestas de los alumnos, improvisar unas acciones por otras, entre otros aspectos. Es de interés estudiar el conocimiento del profesor de matemáticas manifestado en la acción de enseñar, para apreciar su desarrollo profesional y la idiosincrasia de cada profesor. Es especialmente interesante, profundizar en el conocimiento especializado del profesorado para la enseñanza de las matemáticas (CARRILLO et al., 2013); es decir, aquel conocimiento que ponen en juego quienes tienen la intencionalidad de enseñar un contenido matemático.

En esta investigación nos focalizamos en describir el conocimiento especializado de un profesor de matemáticas experto de Educación Primaria, que enseña el tema de los números racionales a estudiantes de 11 a 12 años de edad. Exponemos una descripción detallada del conocimiento que manifiesta un profesor en 21 sesiones de clases, al enseñar el tema de los números racionales. La descripción del conocimiento revelado por el docente nos aproxima a la comprensión del conocimiento especializado del profesor en un entorno real. 
El profesor se ha seleccionado empleando criterios que nos llevan a considerarlo un docente experto, quien puede aportar más información sobre qué conocimiento ha desarrollado a lo largo de su práctica profesional. En lo que sigue, detallamos los aspectos conceptuales en los que se enmarca la investigación, la metodología que nos guía a una descripción del conocimiento del profesor y los resultados a los que hemos llegado.

\section{El conocimiento especializado del profesor de matemáticas}

Una importante contribución al estudio del conocimiento profesional de los profesores aparece en los trabajos de Shulman $(1986 ; 1987)$, en los que se busca resaltar la importancia del conocimiento del contenido para la enseñanza y diferenciarlo del conocimiento del contenido que tienen otros profesionales. Estos fundamentos han inspirado al grupo de investigación de la Universidad de Michigan liderado por Deborah Ball, quienes se centran en el estudio de la naturaleza del conocimiento matemático necesario para enseñar y en el desarrollo de medidas que hacen posible el análisis de relaciones entre el conocimiento matemático para la enseñanza, la calidad de su enseñanza y el rendimiento de los estudiantes (HILL et al., 2008). Ellos establecen una base práctica basada en lo que se denomina conocimiento matemático para la enseñanza (en inglés Mathematical Knowledge for Teaching - MKT ${ }^{1}$ ), que se conceptualiza como "el conocimiento matemático que los profesores utilizan en el aula para producir aprendizaje y crecimiento en los alumnos" (HILL; BALL; SCHILLING, 2008, p.374).

Los estudios de este equipo de investigación han logrado caracterizar en detalle el conocimiento matemático para la enseñanza, basándose en componentes del conocimiento profesional propuesto por Shulman. Plantean un modelo de conocimiento en el que distinguen dos grandes dominios: el conocimiento del contenido matemático y el conocimiento didáctico del contenido.

El conocimiento del contenido matemático está compuesto por tres subdominios. El conocimiento común del contenido, que alude al "conocimiento matemático y habilidades que se emplean en situaciones que no son exclusivas de la enseñanza" (BALL; THAMES; PHELPS, 2008, p.399), incluyendo, entre otros, el conocimiento que el profesor pone en juego para resolver problemas matemáticos, operar correctamente etc. El conocimiento especializado del contenido, definido como el "conocimiento matemático y habilidad

\footnotetext{
${ }^{1}$ Conservamos las siglas en inglés del modelo.
} 
exclusiva para la enseñanza" (BALL; THAMES; PHELPS, 2008, p.400-401). El conocimiento del horizonte matemático es "el conocimiento que tiene el docente de cómo están relacionados los temas matemáticos incluidos en el currículo" (BALL; THAMES; PHELPS, 2008, p.403). Puede considerarse como el conocimiento sobre las relaciones entre los distintos temas matemáticos y la forma en que el aprendizaje de los temas evoluciona en los distintos niveles escolares.

El conocimiento didáctico del contenido queda compuesto por tres subdominios. El conocimiento del contenido y de los estudiantes, que implica el "conocimiento del contenido que se entrelaza con el conocimiento de cómo los estudiantes piensan, saben o aprenden un contenido particular" (HILL et al., 2008, p.375); es decir, es el conocimiento que se utiliza en tareas de enseñanza que conllevan atender a un contenido específico y en aspectos particulares de los alumnos. El conocimiento del contenido y la enseñanza queda definido como "el conocimiento que combina el conocimiento sobre la enseñanza con el matemático" (BALL; THAMES; PHELPS, 2008, p.401); es decir, abarca saber construir procesos pertinentes para tratar de estimular el aprendizaje y corregir los errores y concepciones erróneas de los estudiantes. Finalmente, el conocimiento del currículo alude al conocimiento de los objetivos, contenidos, fines, orientaciones curriculares, materiales y recursos disponibles para la enseñanza, que permiten al profesor guiar su práctica y seleccionar las tareas adecuadas para el aprendizaje de sus estudiantes (BALL; THAMES; PHELPS, 2008).

Los trabajos desarrollados por Ball y colaboradores tienen gran repercusión en Educación Matemática por la extensión de sus estudios y la cualidad analítica de sus propuestas sobre el conocimiento del profesor (BALL, 2000; BALL; THAMES; PHELPS, 2008; HILL et al., 2008). Se trata de un modelo que surge de la observación de la práctica docente y de cuestionarios aplicados a los profesores, y puede emplearse como punto de referencia para la formación del profesor. No obstante, tal como los propios autores han reflejado en sus investigaciones, se requiere una mayor precisión en la naturaleza de cada dominio de conocimiento del profesor necesario para su práctica.

El grupo SIDM de la Universidad de Huelva, España, liderado por José Carrillo, ha precisado algunos aspectos del modelo MKT, considerando el carácter especializado del modelo completo, no exclusivamente de uno de sus subdominios, lo que lleva a redefinir el modelo MKT dando origen al modelo de conocimiento especializado del profesor de 
matemáticas (Mathematics Teacher's Specialized Knowledge - MTSK ${ }^{2}$ ). El modelo MTSK se centra en la especificidad del conocimiento del profesor de matemáticas respecto de la enseñanza del contenido, además considera las creencias de los profesores relacionadas con las matemáticas y la enseñanza de las matemáticas. En este trabajo las creencias no serán objeto de estudio.

El modelo MTSK parte, al igual que el modelo MKT, de dos grandes dominios de conocimiento: (a) conocimiento del contenido matemático $(\mathrm{MK})$ y (b) conocimiento didáctico del contenido (PCK), como se ilustra en la Figura 1.

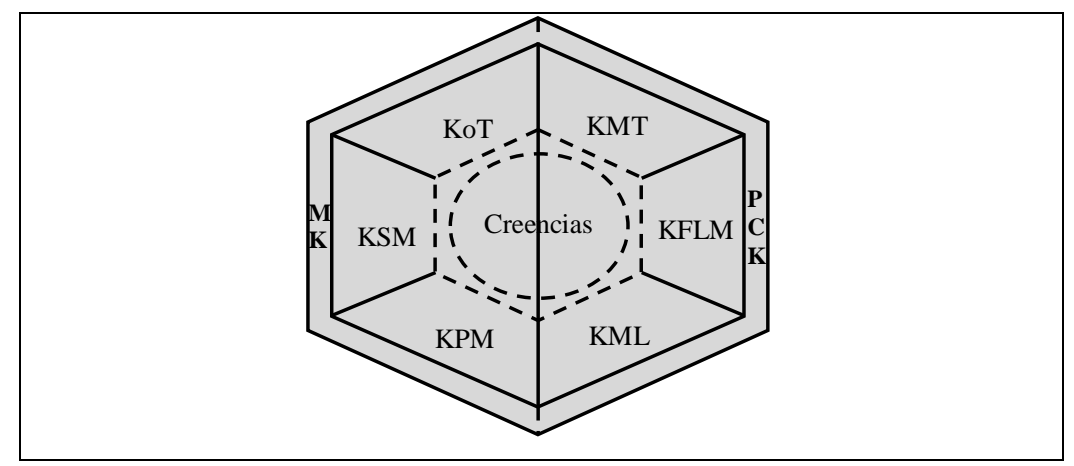

Figura 1- Modelo de Conocimiento Especializado del Profesor de Matemáticas (MTSK)

El conocimiento del contenido matemático (MK) es considerado un elemento articulador del MTSK; asimismo, el conocimiento didáctico del contenido (PCK) se centra en las diferentes formas de profundizar en el contenido matemático cuando se tiene la intención de enseñanza y aprendizaje.

(a) El conocimiento del contenido matemático está compuesto por tres subdominios de conocimiento.

El conocimiento de los temas (Knowledge of Topics - KoT) incluye el conocimiento de los conceptos y procedimientos matemáticos con sus correspondientes fundamentos. Este subdominio se concreta en saber matemáticas; conocer los aspectos fenomenológicos asociados al tema; conocer los distintos significados del tema; conocer ejemplos específicos en aspectos concretos de un tema matemático, etc.

El conocimiento de la estructura de las matemáticas (Knowledge of the Structure of Mathematics - KSM) es el subdominio que encierra una visión de conjunto de la matemática. Considera la idea de conexión y complejidad del contenido matemático. Las conexiones

\footnotetext{
2 Una discusión más amplia del conocimiento especializado del profesor de matemáticas se encuentra en CARRILlO, J.; ClimenT, N.; CONTRERAS, L.C.; MUÑOZ-CATALÁN, M. C. Mathematics Teacher Specialized Knowledge. In: PROCEEDINGS OF CERME 8. CERME 8. 2013. Antalya, Turquía.
} 
abarcan las interconceptuales y temporales ${ }^{3}$ (MARTÍNEZ et al., 2011). Las conexiones interconceptuales comprenden vínculos entre ideas o conceptos matemáticos distintos, siendo los conectores las ideas matemáticas que vinculan representaciones de un concepto con el de otros. Las conexiones temporales enlazan los conocimientos previos y posteriores con el contenido de enseñanza, permitiendo, por ejemplo, estudiar otras propiedades de un concepto o procedimiento en situaciones nuevas o más complejas, como se define en el conocimiento del horizonte del modelo MKT.

La complejidad matemática obliga al docente a analizar los contenidos matemáticos, con el objeto de establecer aquellos que se conectan o son parte de la matemática, lo que implica una comprensión global de la estructura de la matemática vinculada a los conceptos (MONTES et al., 2013).

El conocimiento de la práctica matemática (Knowledge of the Practice of Mathematics - KPM) implica el modo de proceder en matemáticas. Incluye el conocimiento de las formas de conocer y crear o producir en matemáticas (conocimiento sintáctico sobre la lógica en matemáticas), el razonamiento y la prueba, saber definir y usar definiciones, elegir representaciones, argumentar, generalizar o explorar, aspectos de la comunicación matemática (CARRILLO et al, 2013).

Además, el modelo considera los aspectos de enseñanza y de aprendizaje de un contenido matemático, así como las consideraciones curriculares y el conocimiento didáctico derivado de la literatura de investigación, que configuran el dominio del conocimiento didáctico del contenido matemático (PCK).

(b) El PCK está compuesto por tres subdominios de conocimiento:

El conocimiento de la enseñanza de las matemáticas (Knowledge of Mathematics Teaching - KMT) es el conocimiento que permite al profesor elegir una determinada representación o un material para la enseñanza de un concepto o procedimiento, seleccionar ejemplos y tareas matemáticas para el proceso de instrucción; o bien, elegir recursos didácticos que lleven a adquirir, reforzar o ejercitar los contenidos. También, incluye el conocimiento de teorías de enseñanza, como por ejemplo, enseñar a través de la resolución de problemas.

El conocimiento de las características del aprendizaje de las matemáticas (Knowledge of Features of Mathematics Learning - KFLM) es aquel conocimiento que permite al profesor conocer el modo de pensar de los estudiantes sobre las tareas matemáticas y

\footnotetext{
${ }^{3}$ Las conexiones intraconceptuales, que implican enlaces hacia el interior de un mismo concepto, entrarían en el KoT.
} 
comprende todo lo relacionado con generar aprendizaje de las matemáticas. Por ejemplo, conocer las dificultades más frecuentes en el aprendizaje de un contenido o procedimiento matemático, así como saber detectarlas en las respuestas erróneas de los alumnos.

El conocimiento de los estándares de aprendizaje (Knowledge of Mathematics Learning Standards - KMLS) alude a conocer los contenidos propuestos en las normativas curriculares de los niveles de enseñanza (los contenidos, objetivos, orientaciones de enseñanza, los materiales o recursos en los niveles educativos). Puede extenderse a orientaciones que van más allá de las estipuladas en los documentos oficiales; es decir, aquellas recomendaciones de expertos en la materia o asociaciones profesionales o de investigadores (NCTM, PISA, OCDE, UNESCO), investigaciones en el área, entre otros.

Los seis subdominios que dan origen al modelo de Conocimiento Especializado del Profesor de Matemáticas sitúan la matemática como elemento común, centrando la atención en las diferentes formas de profundizar en el contenido matemático cuando es objeto de enseñanza y aprendizaje.

El modelo MTSK se muestra como una herramienta de investigación que permite identificar con precisión los dominios de conocimiento. El grupo de investigación está identificando indicadores precisos de conocimiento para cada subdominio. Ello nos ha permitido establecer un sistema de categorías con el que analizar las clases impartidas por el profesor, como presentamos en el siguiente apartado.

\section{Categorías de los subdominios del conocimiento del profesor}

Para elaborar el sistema de categorías para analizar el conocimiento del profesor nos hemos valido de la realización de un análisis didáctico de la enseñanza y aprendizaje de los números racionales. El grupo de investigación FQM193. Didáctica de la Matemática. Pensamiento Numérico, del Departamento de Didáctica de la Matemática de la Universidad de Granada, ha definido el análisis didáctico como "el procedimiento con el que es posible explorar, profundizar y trabajar con los diferentes y múltiples significados del contenido matemático escolar, para efectos de diseñar, llevar a la práctica y evaluar actividades de enseñanza y aprendizaje.” (GÓMEZ, 2007, p.18-19). Esta idea del análisis didáctico se fundamenta en la teoría curricular que se articula a partir de cuatro dimensiones, cultural/conceptual, cognitiva, ética o formativa y social, en distintos niveles de especificación (RICO; LUPIÁÑEZ; MOLINA, 2013; RICO, 1997a; RICO, 1997b), siendo un recurso que permite al profesor organizar la actividad de enseñanza en relación con un tema 
matemático específico. Así, este análisis tiene por objetivo facilitar la práctica del profesor de matemáticas, de una manera sistemática y profunda, considerando el máximo de dimensiones que influyen en su actuación, mediante cuatro tipos de análisis parciales: a) de contenido, b) cognitivo, c) de instrucción y d) de actuación (GÓMEZ, 2007).

Para profundizar en el conocimiento de la enseñanza de los números racionales hemos realizado el análisis didáctico de los números racionales para tener un referente con el cual comprender mejor los diversos elementos que intervienen en la enseñanza de este contenido (ROJAS; FLORES; CARRILLO, 2013; ROJAS; FLORES; RAMOS, 2013).

Luego de realizar el análisis didáctico del contenido matemático, en nuestro caso de los números racionales, examinamos los componentes que intervienen según la descripción de los subdominios de conocimiento del modelo MTSK. Lo que lleva a establecer categorías respecto de cada subdominio de conocimiento. En el Cuadro 1 indicamos las categorías establecidas para los tres subdominios que abordamos en este trabajo.

\begin{tabular}{|cl|}
\hline & - Conceptos \\
& - Fenomenología \\
KoT & - Procedimientos matemáticos \\
& - Sistemas de representación \\
& - Aspectos de comunicación matemática \\
& - Tareas matemáticas \\
\hline & - Características de aprendizaje \\
KFLM & - Errores y dificultades \\
& - Tareas matemáticas \\
& - Materiales y recursos \\
& - Tareas matemáticas \\
KMT & - Estrategias \\
& - Sistemas de representación \\
& - Formas de uso de los materiales y recursos \\
\hline
\end{tabular}

Cuadro 1- Categorías relacionadas con el análisis didáctico

Para cada categoría establecemos indicadores de conocimientos que nos permiten identificar qué conocimiento se observa a partir de la actuación del profesor en el aula. Hemos definido 57 indicadores respecto de los seis subdominios del modelo de conocimiento MTSK y según las categorías que surgen del análisis didáctico. En el Cuadro 2 detallamos algunos indicadores de conocimiento para el subdominio KoT en relación con nuestro tema de estudio.

\footnotetext{
${ }^{4}$ Las tareas matemáticas se indican en los subdominio KoT, KFLM y KMT y tienen indicadores de conocimiento diferentes respecto de cada dominio. Por ejemplo, las tareas nos permiten ver los significados de las fracciones que maneja el profesor, siendo un aspecto de KoT (aspectos matemáticos); ahora, si el profesor usa criterios explícitos para justificar que la tarea es adecuada al nivel escolar de enseñanza, estaríamos en el KMT.
} 
1. Conocimiento de la estructura conceptual de los números racionales. (Entre otros: la idea de cuerpo conmutativo respecto de la suma y multiplicación, números con representación decimal finita o periódica, construcción formal como conjunto cociente de los enteros, denso en $R$, etc.).

2. Conocimiento de los distintos temas, conceptos y procedimientos matemáticos vinculados a los números racionales. (Entre otros, la proporcionalidad, la probabilidad, razones trigonométricas, semejanza de figuras).

3. Precisión en las definiciones y propiedades y riqueza de relaciones entre los conceptos matemáticos. (Entre otros: que en $Q$ se pueden resolver todas las ecuaciones del tipo $a x=b, a \in Q$ y $b \in Q$, que puede definirse un orden total, etc.).

4. Conocimiento de distintos significados del concepto de fracción (relación parte-todo, reparto, medida, cociente, operador y razón).

5. Conocimiento de campos de utilidad del contenido en ámbitos específicos relacionados con el pensamiento multiplicativo inverso y la proporcionalidad, especialmente si se refiere a contenidos con mayor grado de formalización (por ejemplo, alude a las concepciones de los números racionales como razón, desarrolla el pensamiento proporcional, etc.).

6. Conocimiento de contextos que aparecen en diversas situaciones en las que se aplican los racionales (por ejemplo, medidas de magnitudes -medio kilo, tres cuartos de hora- o relaciones concretas entre cantidades -escala de 1:1000, cartografía; tanto por ciento, comercio, por mil; falta el 10\%, descuento de un $10 \%$, construcción-, entre otros).

Cuadro 2- Indicadores de conocimiento respecto de dos categorías

Los indicadores de conocimiento tuvieron una primera redacción que fue sometida a revisiones de expertos en el área de Didáctica de la Matemática, posteriormente se consideraron las revisiones y observaciones para elaborar la lista final de indicadores.

\section{Metodología}

El objetivo que guía este estudio es describir el conocimiento manifestado por un profesor de Educación Primaria al enseñar el tema de los números racionales. Por lo cual hemos elegido el estudio de caso como diseño de investigación (STAKE, 2007).

Empleamos métodos cualitativos vinculados al paradigma interpretativo. Mediante la observación no participante indagamos, detallada y sistemáticamente, las características de un proceso de enseñanza, de modo de alcanzar el objetivo planteado (COHEN; MANION; MORRISON, 2011). A continuación, describimos la selección del caso y el proceso de recogida y análisis de datos.

\subsection{Selección del caso}

Para seleccionar al profesor informante de la investigación hemos determinado criterios de selección e identificación de profesores expertos. De la revisión de la literatura extraemos diferentes cualidades para seleccionar a docentes expertos, aspectos que fueron 
organizados en características primarias y secundarias (ROJAS; CARRILLO; FLORES, 2012). Las características primarias aluden a cuestiones específicas de la tarea de enseñanza y relacionadas con el conocimiento, como comprender los contenidos específicos y la forma en que aprenden los estudiantes y conocer diversas estrategias de enseñanza, entre otras. Las características secundarias aluden a aspectos objetivos, como ser docente en ejercicio con cinco o más años de experiencia, haber destacado en las evaluaciones institucionales y nacionales (si se aplican), haber enseñado con anterioridad el contenido matemático escolar.

El profesor informante tiene una formación de maestro de Educación Primaria, especialista en matemáticas (tres años de formación como maestro generalista, pero con atención especial a las matemáticas). Ha ejercido la docencia de matemáticas durante 34 años, en la Educación Primaria, participa activamente en sociedades y congresos profesionales de Educación Matemática y constantemente está realizando o guiando cursos de perfeccionamientos en el área de matemáticas. Ha sido recomendado por sus pares al reconocer que domina la materia que enseña. A partir de una entrevista inicial al profesor identificamos que cumple con la totalidad de las características secundarias, por lo que consideramos de interés profundizar en su conocimiento al enseñar el tema de los números racionales.

\subsection{Recogida de datos}

La información sobre la enseñanza puesta en marcha por el profesor y las características de cada la clase ejecutada, se obtiene mediante la observación no participante. Las 21 sesiones, donde se aborda el tema de los números racionales, son grabadas en audio y vídeo. La grabación y el examen de las imágenes se transcriben textuales, complementándose con las notas de campo.

\subsection{Análisis de datos}

El análisis se aplica al texto que registra la actividad matemática desarrollada en las clases. La información de audio y vídeo se transcribe por turnos de intervención, como ilustra la Figura 2, a través del criterio conversacional que consiste en separar las declaraciones o turnos de palabras u oraciones de los sujetos implicados; es decir, cuando interviene el profesor y los estudiantes (RODRÍGUEZ; GIL; GARCÍA, 1996, p. 207). 


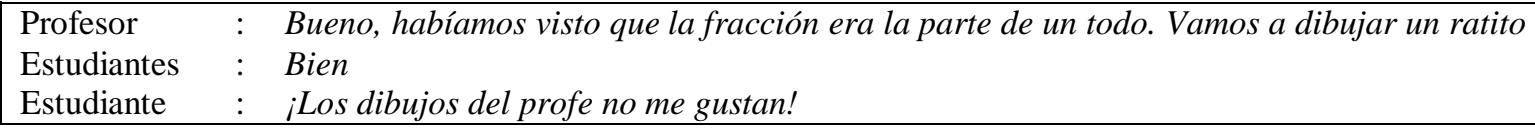

Figura 2- Transcripción por turnos de intervención

Luego, dividimos los datos en episodios que corresponden a fragmentos de intervenciones que tienen una secuencia de acciones y un principio y un fin reconocible (KRIPPENDORFF, 1990, p.85). Los episodios tienen un sentido completo, mostrando, por ejemplo, la ejecución de una tarea o la explicación de un concepto. Posteriormente, en cada episodio vamos identificando fragmentos de información (no necesariamente el episodio completo) que se relacionan con los indicadores de conocimientos establecidos. En una matriz descriptiva, como se ejemplifica en la Figura 3, indicamos cada fragmento (intervalo según un número asignado para cada intervención) que se corresponde con los indicadores de conocimientos definidos.

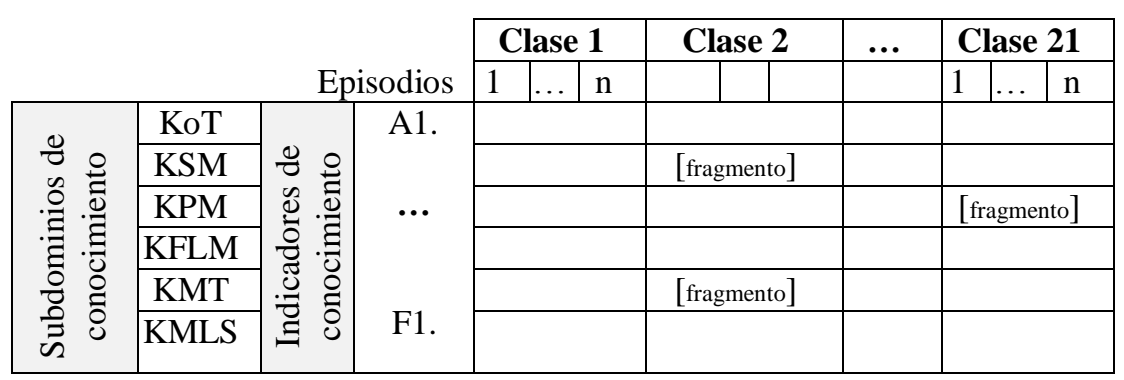

Figura 3- Matriz de registro de las unidades de información

El análisis de los datos se realiza a través de una descripción detallada interpretativa (FOX, 1981). Esto nos permite identificar los indicadores de conocimiento que surgen a lo largo de las 21 clases y describir el conocimiento que manifiesta el profesor.

\section{Conocimiento especializado del profesor al enseñar las fracciones}

En la enseñanza impartida por el profesor de este estudio prevalece una comunicación instructiva en las cuales se integran las contribuciones de los estudiantes (CARRILLO et al., 2008). El profesor es un gestor de las actividades, más que informador de los contenidos. Utiliza, explícitamente, preguntas que llevan a los estudiantes a desarrollar o reforzar ideas matemáticas, aclarar o mejorar aquellas interrogantes que surgen a lo largo del proceso, dejando espacio para que los alumnos reflexionen sobre aspectos del contenido, focalizándose en las ideas o señalamientos de sus estudiantes. Aunque estos aspectos se relacionan con el 
conocimiento pedagógico general, nos llevan a una primera aproximación de la dinámica de enseñanza generada por el profesor.

Detallamos el conocimiento especializado del profesor de matemáticas, respecto de tres subdominios de conocimiento del modelo MTSK, aquellos que a lo largo de las clases han aparecido con más significatividad: conocimiento de los temas matemáticos (KoT), conocimiento de las características del aprendizaje de las matemáticas (KMLS) y el conocimiento de la enseñanza de las matemáticas (KMT).

\subsection{Conocimiento de los temas matemáticos (KoT)}

Respecto del indicador conocimiento de la estructura conceptual identificamos que el profesor trabaja el concepto de número racional aludiendo, principalmente, a su expresión fraccionaria, relacionándola con la expresión decimal procedente de la división entre enteros, por ejemplo, siete quintos, es ¿qué división?, siete entre cinco. Esto le permite emplear como números fraccionarios también aquellos que tienen representante fracciones en las que el numerador es múltiplo del denominador; es decir, las que se corresponden con los enteros $\left(3=\frac{?}{3} ; 12=\frac{?}{3} ; \frac{15}{3} \rightarrow 15 \div 3\right)$. Además, la fracción se reconoce como una porción, que expresa una relación multiplicativa entre el número de partes que forman la porción y el total de las partes consideradas de una unidad.

Para trabajar situaciones de fraccionamiento diferencia la noción de fraccionar y dividir. Identifica la noción de dividir con la acción de partir o separar en partes no necesariamente iguales, mientras que alude a fraccionar para la división igualitaria (partes que representan la misma cantidad). Esta distinción le permite al profesor trabajar la fracción como medida de una parte respecto de una unidad o todo y los significados de fracción partetodo y operador. Asimismo, sin nombrar al conjunto de los números racionales (Q), el profesor trabaja la equivalencia, el orden, las operaciones en Q (suma, resta multiplicación y división) y sus propiedades (de orden, equivalencias notables de Q ).

El significado empleado para introducir el concepto de fracción es el de cociente, aunque como apoyo didáctico prevalecen a lo largo de la enseñanza los significados de fracción como parte-todo y operador; empleándose definiciones menos formales como la fracción es una división, un cociente, la fracción es parte de algo, la fracción es un operador.

La fracción, como parte-todo y operador, se trabaja a partir de resolver distintas tareas de fraccionamiento. Los contextos más frecuentes de las tareas introducidas por el docente 
son los que requieren una partición. Por ejemplo, para el caso de la fracción como parte-todo las tareas comprenden dividir figuras en partes de igual tamaño y de distinta forma, donde se debe identificar cada parte con su representación simbólica. La fracción como operador se trabaja a partir de situaciones como la siguiente: identificar los tres cuartos de sesenta fichas (material concreto), esto lleva a expresar una operación multiplicativa $\left(\frac{3}{4} \cdot 60\right)$ sobre una cantidad (60), obteniéndose una división en tantas partes como indica el denominador (4) y una multiplicación por el número de partes que indica el numerador (3).

Respecto a los procedimientos matemáticos apreciamos que el profesor domina aquellos que enseña, disponiendo de diversos procedimientos para actuar. Por ejemplo, al estudiar la fracción como operador se enuncian distintas tareas de fraccionamiento (de reparto/ medida) que buscan obtener algunas de las partes, donde se conoce: a) la medida del todo y la fracción y la incógnita es la medida de la porción $\left(\frac{a}{b} d e c=x\right)$; b) la medida de la porción y la fracción, y la incógnita es la medida del todo $\left(\frac{a}{b} d e x=d \rightarrow x=\frac{d \cdot b}{a}\right)$; y, c) el todo y la porción y la incógnita es la fracción. Para trabajar la fracción como operador, explica las acciones multiplicativas a partir de la representación gráfica, una división en tantas partes como dice el denominador y la multiplicación por la cantidad de partes del numerador, buscando dar sentido a dichas operaciones.

Trabaja el concepto de número mixto como aquel que tiene una parte entera y una parte fraccionaria, relacionado con la fracción impropia. Muestra dos procedimientos para pasar de fracción impropia a número mixto: a) por medio de un dibujo (representación como parte- todo) y b) como división, la parte entera representa al cociente, el numerador de la fracción al resto y el denominador al divisor; así, multiplicando el divisor por el cociente y sumando el resto obtienen el dividendo, formando la fracción impropia con el dividendo y el divisor. El primer procedimiento lleva a obtener el resultado agrupando o juntando las partes de la figura, proceso que será precursor a la suma de fracciones. El segundo procedimiento permite al profesor relacionar con la división entera. Ambos procedimientos llevan a establecer relaciones entre las operaciones y las acciones.

Para establecer cuándo dos fracciones son equivalentes el profesor presenta los siguientes métodos: a) dibujar y comparar si cada parte o porción representa la misma cantidad (la misma área), b) representar las fracciones en una recta numérica y ver si las medidas coinciden sobre la recta, c) calcular el número decimal correspondiente y compararlos, y d) amplificar o simplificar. La equivalencia de fracciones en a) y b) se trabaja bajo el significado de fracción como parte-todo, la fracción indica la medida de una parte 
respecto de un todo. En c) y d) dos fracciones distintas son equivalentes al generar el mismo resultado.

Para sumar o restar fracciones con igual denominador el profesor da sentido a estas operaciones a partir de su representación figural, como se ilustra a continuación.

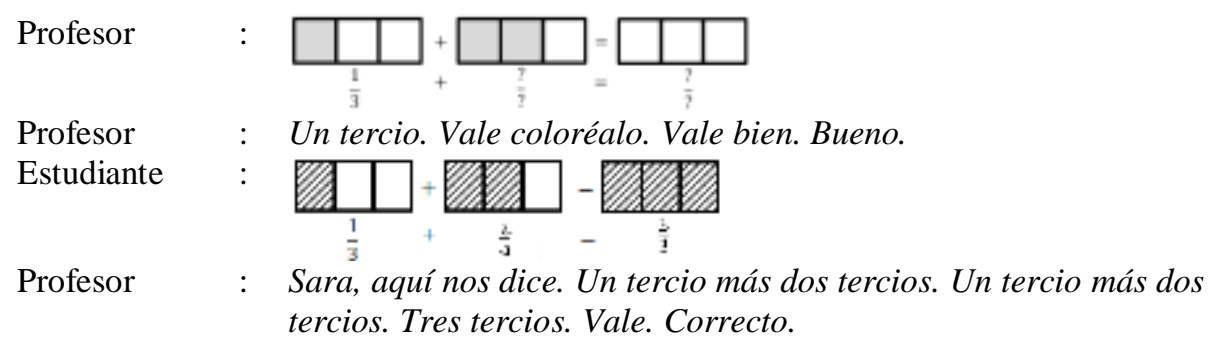

La representación figural permite resolver situaciones de adición o sustracción, donde se tiene una cantidad inicial $\left(\frac{1}{3}\right)$, luego mediante la acción de juntar o quitar otra cantidad $\left(\frac{2}{3}\right)$ se obtiene la cantidad final $\left(\frac{3}{3}\right)$. Posteriormente, suman fracciones con distintos denominadores mediante tres métodos: a) buscando fracciones equivalentes; b) reducción de fracciones a común denominador, por el procedimiento de los productos cruzados; c) calculando el mínimo común múltiplo. Observamos la ordenación lógica que el profesor establece de los procedimientos simbólicos de las operaciones aditivas (suma y resta). Igualmente enfatiza procedimientos numéricos en la multiplicación y división de fracciones, como se refleja en el siguiente fragmento:

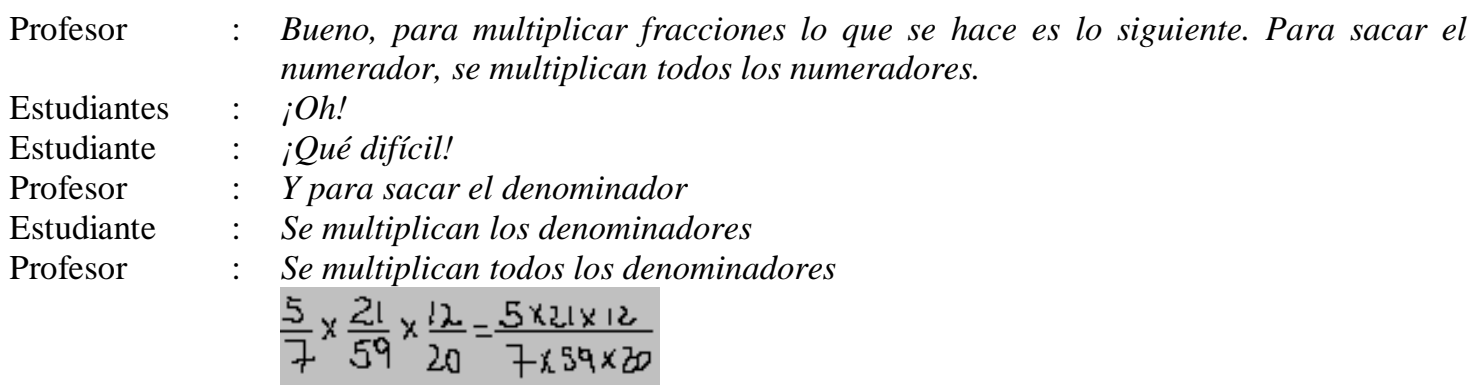

Para multiplicar fracciones lo que se hace es lo siguiente: para sacar el numerador, se multiplican todos los numeradores, y para sacar el denominador, se multiplican todos los denominadores. Para dividir dos fracciones el profesor enuncia que para calcular el numerador de la fracción multiplico el numerador de la primera por el denominador de la segunda y para calcular el denominador, cociente del resultado, multiplico el denominador de la primera por el numerador de la segunda. También emplea la definición formal de esta operación, indicando que dividir dos fracciones es lo mismo que multiplicar la primera 
fracción por la inversa de la segunda fracción. Por tanto, el conocimiento que muestra sobre las operaciones se centra en procedimientos de cálculo, especialmente en la multiplicación y división de fracciones. Mientras que en la suma y resta de fracciones parte de acciones para realizar las operaciones, apoyándose en el empleo de la representación figural (rectángulos) y, luego, llega a expresar la fracción como símbolo, que es el resultado de la acción de juntar o quitar partes de una unidad.

Sobre los sistemas de representación, al trabajar la fracción como parte-todo y operador se emplea mayoritariamente los registros simbólicos (división indicada y decimal), verbal, figural y gráfica. La representación verbal se utiliza para leer las fracciones según su forma simbólica o al enunciar fracciones para escribirlas en su forma simbólica. Al sumar o restar fracciones con igual denominador el profesor emplea la representación figural para dar significado a la acción de sumar y restar, juntando o quitando las partes de una unidad, con lo que muestra los dos pasos de las operaciones, obtención de la porción resultado y asignación de la representación simbólica fraccionaria.

Destacan los modelos basados en representación figural, tanto los discretos como los continuos. Los modelos continuos abarcan diversas magnitudes, prevaleciendo el modelo de área, que adopta una gran riqueza de polígonos, así como formas redondeadas. Conoce y emplea los modelos lineales, tanto en su versión de relación entre segmentos, como la recta numérica, a la que otorga presencia con diversas funciones. La recta numérica le permite representar la fracción como un punto, lo que aprovecha para trabajar el orden y la equivalencia, vinculando con la idea abstracta de número racional. También emplea modelos de volumen, a partir, preferentemente, de prismas rectos rectangulares.

Respecto de la representación material o manipulativa, trabajan con papel (para representar partes congruentes) y con fichas de colores que les permiten el trabajo de la fracción como operador, reforzando situaciones de fraccionamiento como de medida.

Las representaciones y los modelos trabajados en clase permiten al profesor ejemplificar o dar significado a las representaciones verbal y simbólica, asimismo resolver situaciones de fraccionamiento, reforzar los significados de la fracción como parte-todo y como operador y realizar operaciones aditivas. En general, destaca la presencia de la conversión en el sistema de representación figural, sobre todo para sumar fracciones donde los denominadores son iguales $\square \square \cdot \square \square-\square \square$ e ilustrar fracciones equivalentes Asimismo, se pone en juego la conversión entre el sistema de representación simbólico, 
verbal y figural/gráfico en sus modalidades en unidades de longitud y superficie, y en la recta numérica.

Las tareas enunciadas por el profesor son situaciones de reparto y de medida. Las situaciones de reparto son principalmente de partición de un todo y las de medida son por fraccionamiento de la unidad, también presenta algunas situaciones en las que se comparan dos cantidades de una magnitud, estableciendo cuántas veces tiene que ser repetida cada una de ellas para obtener dos cantidades iguales (conmensurabilidad).

\subsection{Conocimiento de las características de aprendizaje de las matemáticas (KFLM)}

Centrándonos en el indicador referente a las características de aprendizaje observamos que en las tareas de fraccionamiento donde intervienen tres datos: dos cantidades (el todo y la parte) y la relación entre ellos (la fracción), el profesor detecta la dificultad que tienen sus estudiantes para identificar estas cantidades en situaciones contextualizadas. Por ejemplo, pide resolver: En una clase se ha votado si se sale o no al recreo cuando llueva, 15 niños han votado que sí y representan tres quintos de la clase. ¿Cuántos niños hay en total en clase? ¿Cuántos prefieren no salir al recreo cuando llueva? En este problema, varios de los estudiantes consideran la porción dada (15 niños) como la unidad (la cantidad de alumnos de la clase). Esto lleva al profesor a solicitar: ¿Por qué no me hacen un dibujo de la clase?. A partir de la representación establecida los alumnos identifican la medida de cada porción o parte (5), y de la porción dada (15) (Manuel, en esos tres quintos, ¿cuántos alumnos hay?) y la unidad o todo (25 niños) (mi pregunta es, si en esos tres quintos hay quince, ¿cuántos hay en toda la clase?).

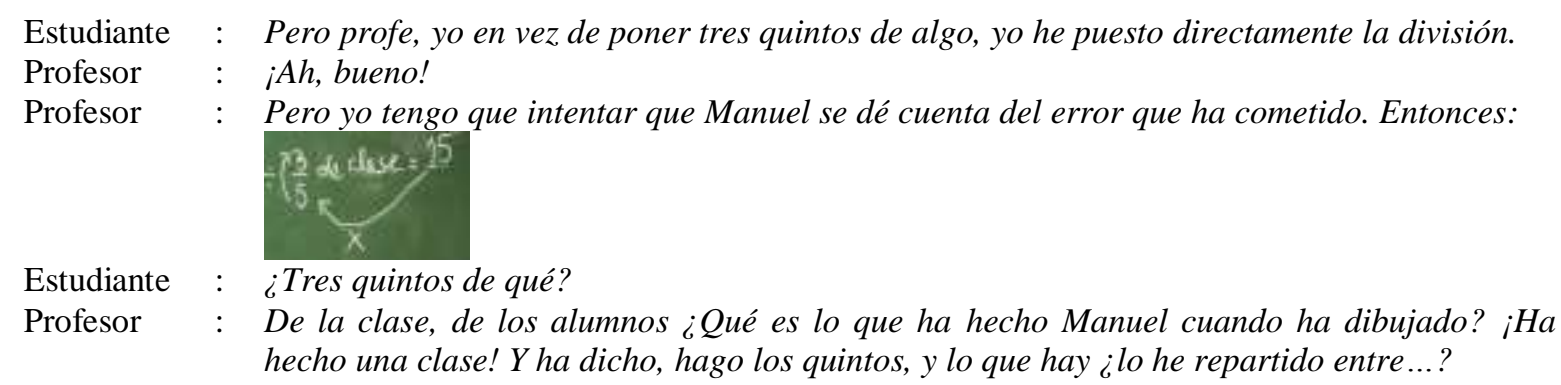

También, en este tipo de situaciones los estudiantes confunden las operaciones implicadas en el fraccionamiento $\left(\frac{3}{5}\right.$ de la clase $=15 \rightarrow 15 \times 3=45$ y $\left.45 \div 5=9\right)$, lo que lleva al profesor a explicitar que aquí habéis cometido uno de los errores que suelen cometer los alumnos, cuando se está calculando la fracción de una cantidad. El profesor orienta a sus 
estudiantes a que representen las cantidades mediante una representación figural, con la que puedan identificar el papel de los términos de la fracción y contrastar la validez de su respuesta.

Vemos que el profesor identifica la imagen de un concepto, que la infiere en base a las respuestas de los estudiantes. Específicamente, conoce las formas en que los alumnos pueden interpretar los conceptos estudiados, o cuando explican un ejercicio o un problema de forma incorrecta; demuestra disposición para trabajar a partir del error, para que los estudiantes den significado a las situaciones.

El profesor identifica distintos errores que los estudiantes presentan al sumar y restar fracciones con numeradores y denominadores distintos. Por ejemplo, a) suman los denominadores y el numerador lo obtiene multiplicando el nuevo denominador por el numerador correspondiente, y b) calculan el mínimo común múltiplo de denominadores y conservan los numeradores:

a)

b)

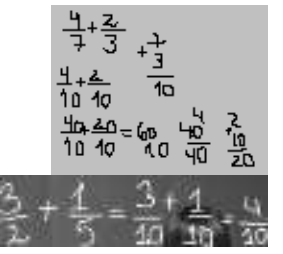

Manuel dice, hay que sumar fracciones que son distintas, [...] ha hecho siete más tres, diez. Luego él se ha acordado que multiplica todos los de abajo menos por el suyo y ha dicho, cuatro por diez cuarenta, dos por diez veinte y pongo el mismo denominador, y ha empezado a sumarlos.

Elena se lo ha estudiado de verdad y ha cogido y ha hecho lo siguiente. [...] Cinco por dos diez, y había puesto ahí el diez, se ve ¿no?

El profesor indica que el estudiante, ejemplo a), ha realizado siete más tres diez. Luego él se ha acordado que multiplica todos los de abajo menos por el suyo y ha dicho, cuatro por diez cuarenta, dos por diez veinte, y pongo el mismo denominador, y ha empezado a sumar. Para el ejemplo b) el profesor enuncia Laura. Ha cogido y ha empezado de la siguiente forma, cinco por dos y ha mantenido los numeradores. Esto nos lleva a establecer que el profesor reconoce los errores comunes que se despliegan al sumar y restar fracciones, por sus expresiones explícitas de que es un error común, como por las estrategias que propone para afrontarlos.

Relacionado con las tareas matemáticas el profesor presenta situaciones que refuerzan los conceptos y procedimientos matemáticos relacionados con las dificultades de aprendizaje de los estudiantes. Asimismo, introduce los contenidos gradualmente, por ejemplo inicia el trabajo con situaciones de fraccionamiento en contextos continuos (área) y va incorporando actividades que involucran contextos discretos como el ejemplo presentado en este apartado. 


\subsection{Conocimiento de la enseñanza de las matemáticas (KMT)}

El profesor presenta un repertorio de tareas de partición de la unidad, como se ilustra en la Figura 4. Para enfatizar la idea de división igualitaria, presenta tareas de dividir diversas figuras en partes de igual tamaño, mediante pasos que atienden a dos aspectos, el tamaño - en relación con alguna magnitud, preferentemente la superficie -, y la forma. Primero, exhibe una actividad donde divide una hoja en dos partes de igual tamaño y forma, luego, una parte la divide en dos partes de igual tamaño y forma, y la otra en trozos de distinto tamaño y forma. La actividad conlleva que cada parte de igual tamaño y forma representa una fracción, mientras las partes de distinto tamaño y forma indican solo una división (en palabras del profesor).

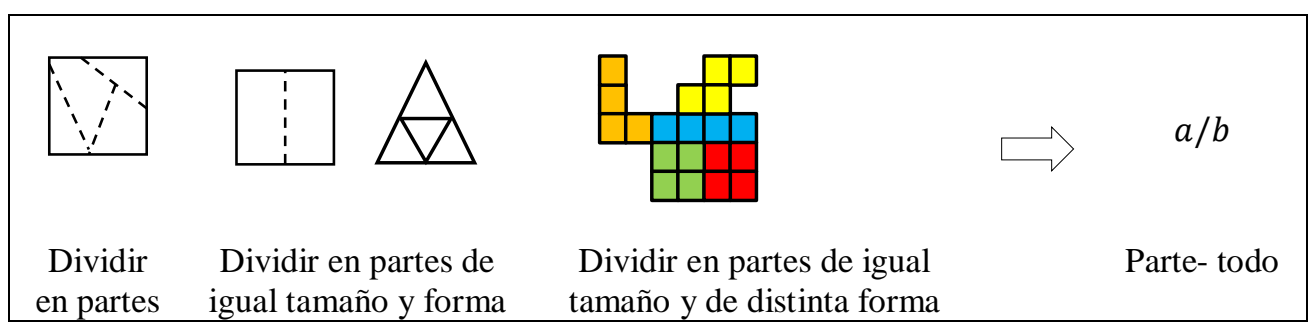

Figura 4- Situaciones de medidas

Continúan el trabajo, dividiendo diversas figuras en partes de igual tamaño y forma, y luego de igual tamaño y distinta forma, con lo que refuerza las nociones de fraccionar y dividir, siendo el fraccionamiento equitativo una de las ideas que presenta dificultad para los estudiantes en diversas situaciones.

Luego, para precisar más la medida se divide la unidad en partes iguales, y si una cantidad de magnitud mide $a / b$ unidades quiere decir que dividiendo la unidad en $b$ partes iguales la cantidad de magnitud a medir equivale a un número $a$ de dichas partes. De este modo expresan cada parte con su representación simbólica (relación existente entre dos cantidades específicas).

La secuenciación de las tareas de fraccionamiento que presenta el profesor es un soporte didáctico para la enseñanza del significado de fracción como parte-todo. Consta de: la acción manipulativa sobre objetos, fraccionamiento, el empleo de representaciones icónicas, y, finalmente, emplea la representación numérica para expresar las acciones anteriores. El proceso seguido por el docente envuelve un conocimiento específico, no solo matemático, sino que integra matemáticas y enseñanza. 
Presenta tareas de fraccionamiento de la unidad en contextos concretos, aludiendo al dinero (euros), permitiendo resolverlas de manera intuitiva, dando sentido a la idea de fracción operador. Se observa, en las primeras sesiones de clase, que las tareas llevan a los estudiantes a relacionar conceptos, procedimientos y darles significados. Sin embargo, en las últimas clases, donde se enseñan las operaciones con fracciones, prevalecen las tareas de reproducción; es decir, se busca que los estudiantes recuerden y demuestren que dominan los procedimientos de cálculo de las operaciones.

Sobre las representaciones, introduce la suma y la resta de fracciones con igual denominador mediante problemas de combinación (solo fracciones con el mismo denominador), que conllevan a juntar o quitar las partes para formar un total (obtener la porción resultado); es decir, tareas en las que subyace un planteamiento directo (conocen las partes o cantidades y se pide el total). Primero emplean la representación figural, luego el trabajo se desvincula de esta representación, centrándose únicamente en los procedimientos de cálculo con las representaciones simbólicas para expresar la porción por medio de una fracción. Comienza obteniendo partes y aplicando fraccionamiento, para luego generalizar por el cálculo simbólico formal.

Los diferentes métodos de cálculo para igualar denominadores antes de sumar o restar fracciones son: a) buscar fracciones equivalentes, b) realizar productos cruzados y c) calcular el mínimo común múltiplo. Aunque se presentan tres procedimientos, prevalece la intención operatoria. Para el caso de la multiplicación y división de fracciones, que envuelven una complejidad conceptual superior a la suma y resta, el trabajo se centra en los procedimientos. Para el caso de la división de fracciones refuerza la regla mnemotécnica de multiplicar en cruz (hacer el dibujito, las líneas que indican el orden de las operaciones).

De los sistemas de representación para la enseñanza observamos que el profesor contantemente emplea la representación verbal, simbólica y figural, y establece relaciones entre ellas. Relacionar las distintas representaciones ayuda a los estudiantes a comprender las tareas, dado que a veces la formulación sólo verbal o simbólica no lleva a una correcta solución. Por ejemplo, en la tarea citada anteriormente [...] 15 niños han votado que sí y representa tres quintos de la clase, ¿cuántos niños hay en total en la clase?, que envuelve el significado de fracción como operador, la mayoría de los estudiantes calcularon $\frac{3}{5}$ de 15 , esto llevó al profesor a solicitar a sus estudiantes que representaran la situación en un dibujo (figura), relacionando distintas representaciones, como se ilustra en la Figura 5. 


\section{Tres quintos de \\ la clase son 15 \\ $\frac{3}{5}$ te $\log =15$}

Figura 5- Representaciones empleadas en clase

A lo largo de las sesiones los materiales y recursos que se emplean son: el papel, para ilustrar si tienen el mismo tamaño las porciones de una figura; fichas de colores (cantidades discretas), para trabajar la fracción como operador (fracción de una cantidad). También el profesor utiliza recursos tecnológicos como las hojas de Excel (función mínimo común múltiplo) que permite calcular el mínimo común múltiplo de varios números y la calculadora. Estas herramientas ayudan a comprobar la validez de los cálculos en los ejercicios que han realizado por otros medios.

En general, en cada clase observamos un repertorio de tareas que permiten introducir o reforzar algún concepto, cambiar de representaciones, reforzar o ejercitar los procedimientos. Podemos inferir que la enseñanza está organizada en las siguientes fases: a) puesta en común de las soluciones de las tareas dejadas en la clase anterior, b) resolución de tareas que implican trabajar un concepto o un procedimiento, c) refuerzo de las ideas básicas, introducción de conceptos o procedimientos, y d) repaso y ejercitación de lo enseñado.

\section{Conclusiones}

Una idea central que surge, del análisis de las sesiones de clase, es que el profesor enseña los números racionales como números fraccionarios. La enseñanza del contenido se desvincula de los aspectos matemáticos formales y se centra en dar sentido a la representación fraccionaria del número racional, según distintos significados. Explícitamente, el profesor trabaja la fracción como parte-todo y operador y, en alguna ocasión, alude al significado de fracción como cociente. Predomina el significado de parte-todo en la conceptualización de la fracción, especialmente en situaciones que llevan a pasar de la representación figural a la simbólica (expresar con una fracción las partes pintadas), complementando con tareas que envuelven trabajar distintas magnitudes (longitud, superficie), identificar la unidad, completar la unidad, entre otras.

El significado de fracción como parte-todo se conecta con el significado de fracción como operador; pese a las diferencias que existen entre ellas (ESCOLANO; GAIRÍN, 2005). Para ello relaciona la expresión simbólica en forma de fracción, con la representación gráfica (representación de la porción en la fracción como parte-todo), con las operaciones de 
multiplicar y dividir por los términos de la fracción, reconociendo la importancia multiplicativa del número racional. Esta acción se realiza en contextos continuos o discretos.

En el caso del fraccionamiento, propone al menos dos formas: $\frac{a}{b}$ de $c \rightarrow(a \cdot c) \div b \mathrm{y}$ $\frac{a}{b}$ de $c \rightarrow a \cdot(c \div b)$.

En las operaciones para multiplicar y dividir fracciones, el profesor se limita a la enseñanza de los procedimientos simbólicos mediante algoritmos convencionales, promueve la automatización de los algoritmos para efectuar las operaciones. Esto nos lleva a establecer que el profesor muestra un buen manejo de los procedimientos matemáticos enunciados para operar correctamente, plantear y resolver situaciones matemáticas, teniendo conocimiento del tema (KoT). Sin embargo, no muestra, ni busca una comprensión profunda del proceso o plantea situaciones en que tengan sentido las operaciones, antes de automatizar los procedimientos. Por ello podemos decir que no busca desarrollar el sentido numérico de las operaciones multiplicativas de los números racionales, ya que escasamente se establecen relaciones numéricas y apenas se aprecian en enunciados que muestren estas acciones; solo se refuerzan los procedimientos.

En las actividades que involucran indagación, exploración o ejercitación el profesor también permite espacios para la reflexión, buscando que los estudiantes modifiquen la comprensión matemática de lo abordado cuando resuelven incorrectamente. Dentro de estas estrategias de enseñanza el profesor refleja conocer los errores y las dificultades que los estudiantes presentan al trabajar el tema matemático, desarrolla explicaciones y enuncia tareas que refuerzan los conceptos o procedimientos matemáticos. Lo que lleva a inferir que el profesor tiene conocimiento de las características de aprendizaje de los números racionales, igualmente de las características de la enseñanza del tema matemático. Esto último, al emplear distintas estrategias de enseñanza (cambio de representaciones, trabajo con material concreto) para abordar los errores y dificultades asociados con el tema, de modo de permitir a los estudiantes adquirir o reforzar el contenido y, principalmente, desarrollar líneas argumentales que faciliten la adquisición de los conceptos y los procedimientos matemáticos estudiados. Los sistemas de representación verbal, simbólico y figural fueron los más usados en la enseñanza del tema, complementándose, en ocasiones, con materiales manipulativos; aspectos necesarios en los primeros años escolares donde se introduce el contenido de los números racionales (CASTRO; TORRALBO, 2008).

En resumen, el análisis de las clases observadas sobre la enseñanza de los números racionales ha puesto de relieve un predominio de situaciones en las que destaca el 
conocimiento didáctico del contenido. De este hecho no inferimos ausencias o lagunas en el dominio del conocimiento matemático (acordes con su formación como maestro generalista), ya que, por un lado, la observación del aula no es la única fuente de la que los investigadores podemos extraer indicios del conocimiento del profesor, y, por otro lado, la ausencia de muestra de conocimiento no significa su carencia. Independientemente de esta elucubración sobre la procedencia o explicación de los resultados en cuanto al predominio de un dominio sobre otro, asunto que es poco tangible, el análisis ha mostrado la importancia de profundizar en situaciones de aula para tratar de comprender mejor los elementos que integran el conocimiento del profesor. Esto nos lleva a seguir en la dirección de comprender el conocimiento especializado del profesor de matemáticas, donde los elementos teóricos empleados son un referente potente para comprender este conocimiento.

\section{Referencias}

BALL, D. L.; THAMES, M. H.; PHELPS, G. Content Knowledge for Teaching: What Makes It Special? Journal of Teacher Education, Las Vegas, v. 59, n. 5, p. 389-407, Nov. 2008.

BALL, D. L. Bridging practices. Intertwining content and pedagogy in teaching and learning to teach. Journal of Teacher Education, Las Vegas, v. 51, n. 3, p. 241-247. May. 2000.

CARRILLO, J.; CLIMENT, N.; CONTRERAS, L.C.; MUÑOZ-CATALÁN, M. C. Mathematics Teacher Specialized Knowledge. In: PROCEEDINGS OF THE EIGHTH CONGRESS OF THE EUROPEAN SOCIETY FOR RESEARCH IN MATHEMATICS, $8^{\text {th }}, 2013$, Antalya. CERME 8, Antalya, Turquía: CERME 8. 2013. p. 2985-2994.

CARRILLO, J.; CLIMENT, N.; GORGORIÓ, N.; PRAT, M.; ROJAS, F. Análisis de secuencias de aprendizaje matemático desde la perspectiva de la gestión de la participación. Enseñanza de las Ciencias, Barcelona, v. 26, n. 1, p. 67-76. Mar. 2008.

CASTRO, E.; TORRALBO, M. Fracciones en el currículo de la educación primaria. En: CASTRO.E (Ed.). Didáctica de la matemática de la educación primaria. España: Síntesis. 2008. p. 285-314.

COHEN, L.; MANION, L.; MORRISON, K. Research methods in education. 5. ed. Londres: Routledge, 2011.

ENGLISH, L. Setting an agenda for international research in mathematics education. In: ENGLISH, L. (Ed.). Handbook of international research in mathematics education. New York: Routledge, 2008. p. 3-19.

ESCOLANO, R.; GAIRÍN, J. M. Modelos de medida para la enseñanza de números racionales en educación primaria. UNIÓN Revista Iberoamericana de Educación Matemática, Reston VA, v.1, n. 1, p.17-35. Apr. 2005.

FOX, D. J. El proceso de investigación en la educación. Pamplona: Eunsa.1981. 
GÓMEZ, P. Desarrollo del conocimiento didáctico en un plan de formación inicial de profesores de matemáticas de secundaria. 2007. 911f. Tesis (Doctorado en Matemáticas con especialidad en Didáctica de la Matemática) - Facultad de Educación, Universidad de Granada. España, 2007.

HILL, H. C.; BALL, D. L.; SCHILLING, S. G. Unpacking pedagogical content knowledge: conceptualizing and measuring teachers' topic-specific knowledge of students. Journal for Research in Mathematics Education, Reston VA, v. 39, n. 4, p. 372- 400, July. 2008.

HILL, H. C.; BLUNK, M. L.; CHARALAMBOUS, C. Y.; LEWIS, J. M.; PHELPS, G. C., SLEEP, L.; BALL, D. L. Mathematical knowledge for teaching and the mathematical quality of instruction: An exploratory study. Cognition and Instruction, Mahwah, New Jersey, v. 26, n. 4, p. 430-511, July. 2008.

KRIPPENDORFF, K. Metodología del análisis de contenido. Barcelona, España: Paidós Ibérica.1990.

MARTÍNEZ, M.; GINÉ, C.; FERNÁNDEZ, S.; FIGUEIRAS, L.; DEULOFEU. J. El conocimiento del horizonte matemático: más allá de conectar el presente con el pasado y el futuro. En: SIMPOSIO DE LA SOCIEDAD ESPAÑOLA DE INVESTIGACIÓN EN EDUCACIÓN, Ciudad Real. Anales... Ciudad Real. España, 2011. p. 429-437.

MONTES, M. A.; AGUILAR, A.; CARRILLO, J.; MUÑOZ-CATALÁN, M. C. MTSK: from Common and Horizon Knowledge to Knowledge of Topics and Structures. In: PROCEEDINGS OF THE EIGHTH CONGRESS OF THE EUROPEAN SOCIETY FOR RESEARCH IN MATHEMATICS, $8^{\text {th }}$, Antalya. Proceedings... Antalya, Turquía, 2013. p. 3185-3194.

OCDE. Teachers for tomorrow's schools. Analysis of the world education indicators. Executive Summary. Organization for economicdge of topics and structures. In: PROCEEDINGS OF CERME 8. $8^{\text {th }}$, Antalya. Proceedings... Antalya, Turquía: World Education Indicators Programme, París, Francia. 2001.

RIBEIRO, C. M. Conhecimento matemático para ensinar: uma experiência de formação de professores no caso da multiplicação de decimais. Bolema, Rio Claro, SP, v. 22, n. 34, p. 1-26. dez. 2009.

RICO, L. Bases teóricas del currículo de matemáticas en educación secundaria. Madrid: Síntesis.1997a.

RICO, L. La enseñanza de las matemáticas en la educación secundaria. Barcelona, Horsori. 1997b.

RICO, L.; LUPIÁÑNEZ, J. L.; MOLINA, M. El análisis didáctico en educación matemática.

Metodología de investigación, innovación curricular y formación de profesores. Granada, España: Universidad de Granada. 2013.

RODRÍGUEZ, G.; GIL, J.; GARCÍA, E. Metodología de la investigación cualitativa. Málaga: Aljibe.1996.

ROJAS, N.; FLORES, P.; CARRILLO, J. Caracterización del conocimiento matemático para la enseñanza del concepto de fracción: un estudio de caso. Avances de Investigación en Educación Matemática. España, v. 4, n. 4, p. 47- 64, Oct. 2013.

ROJAS, N.; FLORES, P.; RAMOS, E. El análisis didáctico como herramienta para identificar conocimiento matemático para la enseñanza en la práctica. En: RICO, L.; LUPIÁÑEZ, J.L.; MOLINA. M (Ed.). El análisis didáctico en educación matemática. Metodología de investigación, innovación curricular y formación de profesores. Granada, España: Universidad de Granada. 2013. 
ROJAS, N.; CARRILLO, J.; FLORES, P. Características para identificar a profesores de matemáticas expertos. En: SIMPOSIO DE LA SOCIEDAD ESPAÑOLA DE INVESTIGACIÓN EN EDUCACIÓN, 16., 2012, Jaén. Investigación en Educación Matemática XVI. Jaén. España: SEIEM. 2012. p.479-485.

SCHOENFELD, A. H.; SRIRAMAN, B.; ENGLISH, L. Theories of mathematics education: seeking new frontiers. In: SRIRAMAN, B.; ENGLISH, L (Ed.). Theories of mathematics education.

Seeking new frontiers. Berlin/Heidelberg: ZDM. Mathematics Education. (Springer series: advances in mathematics education). 2010. p. 503-506.

SHULMAN, L. S. Knowledge and Teaching: foundations of the New Reform. Harvard Educational Review, Harvard, v. 57, n. 1, p. 1-22, Feb. 1987.

SHULMAN, L. S. Those Who Understand: Knowledge growth in Teaching. Educational Researcher, Washington, v. 15, n. 2, p. 4-14, Feb. 1986.

STAKE, R. Investigación con estudio de casos. 4. Ed. Madrid: Morata, S. L. 2007.

Submetido em Abril de 2014. Aprovado em Julho de 2014.

\section{ERRATA}

No artigo "Conocimiento Especializado de un Profesor de Matemáticas de Educación Primaria al Enseñar los Números Racionales" publicado no periódico Bolema, 29 (51):143166, na página 143 , onde se lia:

"Máster en Didáctica de la Matemática, Universidad de Granada (UGR), España. Becaria de doctorado Beca hile, Comisión Nacional de Investigación Científica y Tecnológica, Gobierno de Chile en el Departamento de Didáctica de la Matemática de la Universidad de Granada (UGR), Granada, España. Dirección postal: Facultad de Educación, Campus Cartuja, 18071, Granada, España. E-mail: nielka001@gmail.com.”

\section{leia-se:}

"Doctor en Matemáticas, Universidad de Granada (UGR), España. Profesor de la Escuela de Educación, Universidad Católica del Norte (UCN), Antofagasta, Chile. Dirección postal: Angamos 0610. Antofagasta, Chile.E-mail: nrojas03@ucn.cl." 